\title{
VIRAL SUPPRESSION AND DRUG RESISTANCE AMONG HIV POSITIVE WOMEN AT HIGH RISK OF SEXUALLY-TRANSMITTED INFECTIONS ON ANTIRETROVIRAL THERAPY IN KAMPALA, UGANDA
}

Gertrude Namale1, Onesmus Kamacooko', Daniel Bagiire', Yunia Mayanja1, Andrew Abaasa', William Kilembe ${ }^{2}$, Matt A Price $^{3,4}$, Deogratius Ssemwanga, Sandra Lunkuse1, Maria Nanyonjo ${ }^{1}$, William Ssenyonga ${ }^{1}$, Robert Newton ${ }^{1,5}$, Pontiano Kaleebu ${ }^{1}$, Janet Seeley ${ }^{1,6}$

${ }^{1}$ MRC/UVRI and LSHTM Uganda Research Unit, Entebbe Uganda, ${ }^{2}$ Zambia Emory HIV Research Project, Lusaka, Zambia , ${ }^{3}$ International AIDS Vaccine Initiative, New York, USA , ${ }^{4}$ University of California at San Francisco, California, USA, ${ }^{5}$ University of York, UK, ${ }^{6}$ London School of Hygiene and Tropical Medicine, London, UK

\section{AIM}

We aimed to assess prevalence and risk factors for unsuppressed viral load (VL) among HIV positive Female sex workers (FSW) on antiretroviral therapy (ART) in Kampala, Uganda.

\section{METHODS}

We conducted a cross-sectional study between January 2015 - December 2016 using routinely collected data at a research clinic providing services to women at high-risk. Plasma samples from women aged $\geq 18$ years, on ART, were tested at least 6 months after ART initiation for HIV VL. Samples from FSW with unsuppressed VL ( $\geq 1000$ copies $/ \mathrm{mL})$ were tested for HIV drug resistance by sequencing of partial polymerase gene. Sequences were submitted to Stanford University HIV Drug Resistance database analysis program and drug resistance mutations (DRM) identified using the $2009 \mathrm{WHO}$ mutations list. Socio-demographic, sexual behaviour, clinical and VL data was extracted from records at the clinic. We used logistic regression to identify factors associated with unsuppressed VL.

\section{RESULTS}

Four hundred and thirty- two HIV positive women were included; mean age was 32.5 years $(\mathrm{SD}=6.5)$. Of these, $38(9.0 \%)$ had unsuppressed VL. Of these, $94.7 \%(36 / 38)$ were retrieved for testing. Resistance testing was available for $78 \%$ $(28 / 36)$, of which $82.1 \%(23 / 28)$ had at least one major DRM, most frequently M184V (70\% [16/23]) and K103N (65\% [15/23]). We observed a high frequency of Thymidine Analogue Mutations - 65.2\% (15/23). Unsuppressed VL was associated with participant age [young age (18-24)] adjusted odds ratio $(\mathrm{aOR})=5.3(95 \% \mathrm{Cl}$ : 1.6-17.9), self-reported ART adherence [nonadherence] aOR $=2.6(95 \% \mathrm{Cl}: 1.2-5.8), \mathrm{CD} 4+$ T-cell counts $\left[\leq 350\right.$ cells $\left./ \mathrm{mm}^{3}\right]$ aOR $=3.1(95 \%$ $\mathrm{Cl}: 1.4-7.0)$.

ACKNOWLEDGEMENT: UK Medical Research Council (MRC) and the UK Department for International Development (DFID) under the MRC/DFID Concordat agreement which is also part of the EDCTP2 programme supported by the European Union and International AIDS Vaccine Initiative (IAVI) for the funding. The clinic services and ART programme supported by PEPFAR.

\section{CONCLUSIONS ANS RECOMMENDATIONS}

Prevalence of unsuppressed VL was low; however, we found a high proportion of DRMs among the women with unsuppressed VL. Younger age, non-adherence and low CD4+ T-cell counts $(\leq 350)$ increased the odds of unsuppressed VL. Interventions targeting young women to improve viral suppression should be considered.

Table 1: Characteristics of HIV+ FSW and association with unsuppressed viral load in Kampala, Uganda

\begin{tabular}{|c|c|c|c|c|c|}
\hline Variables & $\begin{array}{l}\text { Suppressed } \\
\text { (n (row \%)) }\end{array}$ & $\begin{array}{r}\text { Not } \\
\text { Suppressed } \\
(\mathrm{n}(\text { row \% }))\end{array}$ & $\begin{array}{l}\text { uOR } \\
(95 \% \\
\text { Cl) }\end{array}$ & $\begin{array}{l}\text { LRT } \\
\text { p- } \\
\text { value }\end{array}$ & $\begin{array}{r}\text { aOR(95 } \\
\% \text { Cl) }\end{array}$ \\
\hline Overall (\%) & $394(91)$ & $38(9)$ & & & \\
\hline Age category & & & & 0.064 & \\
\hline $35-54$ & $142(94)$ & $9(6)$ & 1 & & \\
\hline $25-34$ & $216(91)$ & $21(9)$ & $\begin{array}{c}1.5(0.7- \\
3.4)\end{array}$ & & $\begin{array}{r}1.9(0.8- \\
4.7)\end{array}$ \\
\hline $18-24$ & $36(82)$ & $8(18)$ & $\begin{array}{c}3.5(1.3- \\
9.7)\end{array}$ & & $\begin{array}{r}5.3(1.6- \\
17.9)\end{array}$ \\
\hline $\begin{array}{l}\text { Level of } \\
\text { education }\end{array}$ & & & & 0.048 & \\
\hline No education $†$ & $34(85)$ & $6(15)$ & 1 & & \\
\hline $\begin{array}{l}\text { Primary } \\
\text { education }\end{array}$ & $236(94)$ & $15(6)$ & $\begin{array}{c}0.4(0.1- \\
1.0)\end{array}$ & & $\begin{array}{r}0.4(0.1- \\
1.1) \\
\end{array}$ \\
\hline $\begin{array}{l}\text { Secondary and } \\
\text { above }\end{array}$ & 124(88) & $17(12)$ & $\begin{array}{c}0.8(0.3- \\
2.1)\end{array}$ & & $\begin{array}{r}0.9(0.3- \\
2.5)\end{array}$ \\
\hline $\begin{array}{l}\text { Physical violenc } \\
\text { partners in past }\end{array}$ & $\begin{array}{l}\text { e with sexual } \\
\text { three months }\end{array}$ & & & 0.464 & \\
\hline Not & $137(93)$ & $11(7)$ & 1 & & \\
\hline Yes & $257(90)$ & $27(10)$ & $\begin{array}{c}0.8(0.4- \\
1.6)\end{array}$ & & \\
\hline $\begin{array}{l}\text { CD4+ T-cell } \\
\text { counts } \geq 6 \\
\text { months after } \\
\text { ART initiation }\end{array}$ & & & & 0.01 & \\
\hline$>350$ cells $/ \mathrm{mm}^{3}$ & $231(94)$ & $14(6)$ & 1 & & 1 \\
\hline$\leq 350$ cells $/ \mathrm{mm}^{3}$ & 163(87) & $24(13)$ & $\begin{array}{c}2.4(1.2- \\
4.8)\end{array}$ & & $\begin{array}{r}3.1(1.4- \\
7.0)\end{array}$ \\
\hline $\begin{array}{l}\text { Place of ART } \\
\text { initiation }\end{array}$ & & & & 0.072 & \\
\hline $\begin{array}{l}\text { Clinic } \\
\text { initiated } †\end{array}$ & $345(92)$ & $29(8)$ & 1 & & \\
\hline Transfer-in & $49(84)$ & $9(16)$ & $\begin{array}{c}2.2(1.0- \\
4.9)\end{array}$ & & $\begin{array}{r}1.7(0.5- \\
5.9) \\
\end{array}$ \\
\hline $\begin{array}{l}\text { Adherence } \\
\text { status }\end{array}$ & & & & 0.019 & \\
\hline Adherent $†$ & $333(93)$ & $26(7)$ & 1 & & \\
\hline $\begin{array}{l}\text { Self-reported } \\
\text { non adherent }\end{array}$ & $61(84)$ & $12(16)$ & $\begin{array}{c}2.5(1.2- \\
5.3)\end{array}$ & & $\begin{array}{r}2.6(1.2- \\
5.8)\end{array}$ \\
\hline $\begin{array}{l}\text { Duration of } \\
\text { ART (in years) } \\
\text { median (IQR ) }\end{array}$ & $2.3(1.8-3.1)$ & $2.7(2.2-3.8)$ & $\begin{array}{c}1.2(1.0- \\
1.4)\end{array}$ & 0.071 & $\begin{array}{r}1.1(0.8- \\
1.4)\end{array}$ \\
\hline
\end{tabular}

